\title{
Análisis del estrato arbóreo de agroecosistemas en una zona de transición ecológica
}

\author{
Analysis of trees in agroecosystems in an area of ecological transition \\ Laura White-Olascoaga $^{1 *}$, Cristina Chávez-Mejía ${ }^{2}$, David García-Mondragón ${ }^{1}$ \\ ${ }^{1}$ Universidad Autónoma del Estado de México. Facultad de Ciencias. Campus El Cerrillo Piedras Blancas. Carretera \\ Toluca-Ixtlahuaca Km. 14.5, Estado de México, México \\ ${ }^{2}$ Universidad Autónoma del Estado de México. Instituto de Ciencias Agropecuarias y Rurales. Carretera Toluca-Ixtlahuaca Km \\ 14.5 San Cayetano Toluca, Estado de México. \\ ${ }^{*}$ Autor de correspondencia: laurawhiteo@yahoo.com.mx
}

Artículo científico recibido: 02 de septiembre de 2015 aceptado: 01 de junio de 2016

RESUMEN. Los huertos familiares son agroecosistemas heterogéneos en estructura y manejo, en ellos se mantienen procesos de conservación in situ de la biodiversidad. La flora en los huertos es variada, su estudio contribuye a comprender las especies que coexisten y se conservan, además de entender la estructura, importancia, y funciones para las familias de las comunidades rurales. El estudio se enfocó al estrato arbóreo del agroecosistema huerto familiar (AHF) en San Nicolás, Malinalco, México, por tener especies clave en la estructura de los agroecosistemas y por su importancia social, cultural y económica. Se estudiaron 14 agroecosistemas, ubicados en la zona de transición ecológica del Estado de México. El estudio se llevó a cabo de marzo de 2011 a abril de 2012, por medio de entrevistas no estructuradas a los propietarios de los huertos. El objetivo fue realizar un análisis del estrato arbóreo sobre la diversidad, estructura, función y análisis biogeográfico. Los agroecosistemas poseen una diversidad de 36 especies, 26 géneros y 18 familias de plantas. La especie más frecuente fue Persea americana Mill. var. Hass presente en el $100 \%$ de los agroecosistemas. De las especies encontradas, 52.7 \% presentan una distribución natural en México, y las otras son introducidas. aprovechamiento del estrato arbóreo, es con fines de autoconsumo, principalmente como fruta. La diversidad florística de las especies arbóreas es alta, lo que permite ubicar a estos agroecosistemas como importantes sitios de conservación de germoplasma in situ.

Palabras clave: Biogeografía, conocimiento tradicional, diversidad florística, huertos familiares, medicina tradicional

ABSTRACT. Home gardens are heterogeneous agroecosystems in structure and management, in which in-situ biodiversity conservation processes are maintained. The flora in the gardens is varied; their study contributes to understanding the species that coexist and are conserved, in addition to understanding the structure, importance, and functions for families in rural communities. The study focused on the tree stratum of the home garden agroecosystem (HGA) in San Nicolás, Malinalco, Mexico, because it has key species in the structure of agroecosystems and because of its social, cultural and economic importance. Fourteen agroecosystems located in the ecological transition area of the State of Mexico were studied. The study was conducted from March 2011 to April 2012, through unstructured interviews with garden owners. The objective was to conduct an analysis of the tree stratum in terms of diversity, structure, function and biogeography. The agroecosystems have a diversity of 36 species, 26 genera and 18 plant families. The most common species was Persea americana Mill. var. hass present in $100 \%$ of the agroecosystems. Of the species found, $52.7 \%$ have a natural distribution in Mexico, and the others are introduced. The tree stratum is used for purposes of self-consumption, mainly as fruit. The floristic diversity of the tree species is high, which allows categorizing these agroecosystems as important in-situ germplasm conservation sites.

Key words: biogeography; traditional knowledge; floristic diversity; home gardens; traditional medicine. 


\section{INTRODUCCIÓN}

Los huertos familiares son agroecosistemas ampliamente distribuidos en México y el resto del mundo. Generalmente están establecidos anexos a la vivienda, varían en tamaño, de ellos las familias obtienen alimento y en muchos casos son fuente secundaria de ingresos económicos (Mariaca 2012). En ellos se practica la agricultura tradicional caracterizada por utilizar policultivos con diversas especies de plantas arbóreas, arbustivas y herbáceas (Juan et al. 2007, Moctezuma 2010), con interacción con el medio físico y biológico que resulta en un funcionamiento similar a un ecosistema con características intermedias entre un ecosistema natural y uno antrópico (Juan 2014). En los agroecosistemas huertos familiares (AHF) se presentan diversos doseles con funciones específicas. Las especies arbóreas, proporcionan sombra y hojarasca generando microclimas que contribuyen al reciclaje de nutrimentos y al mantenimiento de la fertilidad del suelo; en estratos inferiores, las especies arbustivas y herbáceas evitan la pérdida de suelo debida a la erosión y aumentan la captación de agua por infiltración (Martínez y Juan 2005, Juan et al. 2007).

Las especies arbóreas son fundamentales en la estructura de los AHF (Gliessman 2002), incrementan la diversidad y abundancia de especies y cumplen funciones ambientales, sociales, culturales, económicas, alimenticias, ornamentales, medicinales, entre otros (Hylander y Nemomisa 2008). En algunos casos, especies arbóreas relevantes en el huerto, tanto comercialmente como por su importancia nutrimental tienden a ser preferidas y conservadas, lo que influye en que el conocimiento tradicional de su uso y manejo se mantenga de generación en generación (Kristensen y Lykke 2003). Los usos y valores atribuidos a ciertas especies de árboles contribuyen a su conservación, incluso en áreas naturales protegidas, lo que muestra cómo el uso contribuye en su conservación (Gunnar et al. 2004). El uso y manejo de los huertos, permite la diversidad de especies, tanto cultivadas como no cultivadas (High y Shackleton 2000, Hylander y Nemomissa 2008).
El estudio del estrato arbóreo de los huertos familiares contribuye a documentar procesos de conservación de la diversidad biológica in situ, su uso y manejo, sobre especies cultivadas y no cultivadas, así como la pérdida o deterioro de hábitats (Kristensen y Lykke 2003). El estudio del uso, valor cultural y económico de los diversos productos obtenidos de los árboles, en particular de las especies nativas, permite conocer el manejo y proponer alternativas de conservación para mantener el aprovisionamiento local de bienes (Gunnar et al. 2004). Por lo anterior, el presente estudio tiene el objetivo de analizar el estrato arbóreo en huertos familiares de San Nicolás, municipio de Malinalco, Estado de México.

\section{MATERIALES Y MÉTODOS}

\section{Área de estudio}

La localidad de San Nicolás en el municipio de Malinalco, Estado de México, se ubica en una zona de transición ecológica, geográficamente delimitada por las coordenadas $18^{\circ} 58^{\prime} 22^{\prime \prime}$ LN y $99^{\circ} 29^{\prime} 42^{\prime \prime}$ LO. Tiene una altitud promedio de $1960 \mathrm{msnm}$ y se ubica en el subtrópico del Altiplano Mexicano, en la confluencia de las provincias fisiográficas de la Sierra Madre del Sur y el eje Volcánico, que en su mayor parte están en la subprovincia de Sierras y Valles Guerrerenses. El clima es semicálido, subhúmedo, con lluvias en verano, con temperatura media anual de $20.4^{\circ} \mathrm{C}$. La precipitación pluvial anual promedio es de $1177 \mathrm{~mm}$ con un máximo de 260 a $270 \mathrm{~mm}$ en agosto y mínima de $10 \mathrm{~mm}$ de diciembre a febrero (INEGI 2009).

\section{Muestreó y análisis de datos}

El método incluyó un diagnóstico sobre la riqueza del estrato arbóreo en los agroecosistemas huerto familiar. Se estudiaron catorce sistemas seleccionados por la disposición de los dueños a colaborar en el estudio, a quienes se aplicaron entrevistas no estructuradas preliminares, para el acercamiento a la comunidad y la obtención de información inicial. La elección de los huertos e informantes fue por aceptación a colaborar en el estudio y por 
contar con huerto familiar. Entre marzo de 2011 y abril de 2012 se observaron 14 huertos en 25 recorridos en los que se aplicaron entrevistas, observación directa y muestreo de las especies arbóreas.

El inventario florístico incluyó la recolección de especímenes arbóreos cultivados y silvestres con sus estructuras reproductoras, cada uno se procesó de acuerdo con Lot y Chiang (1986) para su identificación botánica. Para el análisis biogeográfico se recopiló información mediante consulta de la literatura especializada sobre las especies nativas, endémicas e introducidas en el territorio México. Las especies introducidas se ubicaron de acuerdo a su origen geográfico en Asia, Europa y América.

\section{RESULTADOS}

\section{Diversidad florística}

La diversidad florística del estrato arbóreo en los AHF se compone de 36 especies, pertenecientes a 26 géneros y 18 familias. La familia Rutaceae, corresponde el $13.89 \%$ de los ejemplares colectados, la cual presenta cinco especies del género Citrus sp (Tabla 1). En orden de importancia le siguen las familias Fabaceae, Rosaceae y Moraceae con cuatro especies cada una. Estas cuatro familias en conjunto, incluyen el $47.22 \%$ del total de especies presentes en los sistemas. La importancia de estas familias botánicas en los AHF radica en la comercialización de sus frutos en mercados locales y para consumo familiar, por lo que las familias disponen de fruta para consumo en fresco y para la venta, además de que los excedentes los consumen en mermelada o almíbar. La mayoría de los árboles en los huertos son cultivados (94.4\%), los cuales han sido cuidados por generaciones. También hay especies silvestres como Ficus petiolaris Kunth (ornamnetal) y Byrsonima crassifolia (L.) Kunth (alimenticia) que son toleradas por su utilidad, de manera que al establecer el huerto no se remueven, pasando a formar parte de las especies útiles y del agroecosistema.

\section{Estructura}

Los AHF de San Nicolás, son generalmente de forma cuadrangular (85.7\%) o irregular (14.2 $\%$ ), con un área de entre 432 y $5001 \mathrm{~m}^{2}$, en los cuales la familia hace uso y manejo del estrato arbóreo, con base en su conocimiento tradicional, para la obtención a lo largo del año de diversos productos y servicios que apoyan la economía, salud y alimentación de la familia, aunque también son útiles desde el punto de vista estético y espiritual. La estructura vertical y horizontal de los huertos está compuesta por tres estratos bien definidos y relacionados, los cuales son arbóreo, arbustivo y herbáceo, los cuales están bajo un arreglo al azar, lo que permite cubrir los requerimientos de luz, sombra, ventilación y humedad para las especies cultivadas, fomentadas y toleradas de los tres estratos.

En los AHF, existe la presencia de especies arbóreas que dan fisonomía y estructura, y proporcionan al sistema sus características físicas y funciones. Los árboles mantienen la cadena trófica en el sistema, debido a que poseen diferentes fenologías, contando con una producción de flores y frutos a lo largo del año, esto permite el soporte de alimento y refugio para diferentes especies de insectos y aves, especies pilares o clave, las cuáles son Persea americana Mill. var. Hass, y el género Citrus sp., especies que se encuentran en el $100 \%$ de los huertos, Psidium guajava L. en el $78.5 \%, P$. americana Mill. var. Drymifolia (Schltdl. \& Cham) Blake en el $71.4 \%$ y Eriobotrya japonica (Thunb.) Lindl. e Inga jinicuil Schltdl en el $57.14 \%$ de los AHF.

\section{Conocimiento tradicional y uso}

El conocimiento tradicional que las familias poseen sobre la apropiación de su medio, permite el uso diverso del estrato arbóreo, el cual es un recurso importante para el desarrollo y bienestar de la familia, por lo que cada una de las especies satisface diferentes necesidades y posee diferentes funciones (Figura 1).

Uso alimenticio. Los árboles son un componente integral de los sistemas, sembrados y tolerados en los huertos familiares como soporte alimenticio, el $42.62 \%$ del estrato es aprovechado para este 
Tabla 1. Especies arbóreas en huertos familiares en San Nicolás, México.

\begin{tabular}{|c|c|c|}
\hline Nombre común & Nombre científico & Familia \\
\hline Yuca & Yucca filifera Chabaud & Agavaceae \\
\hline Mango & Mangifera indica $\mathrm{L}$. & Anacardiaceae \\
\hline Ciruela criolla & Spondias purpurea $\mathrm{L}$. & Anacardiaceae \\
\hline Chirimoya & Annona cherimola Mill. & Annonaceae \\
\hline Anona & Annona reticulata $\mathrm{L}$. & Annonaceae \\
\hline Ayoyote, codo fraile & Thevetia thevetioides (H.B.K.)Schum. & Apocynaceae \\
\hline Araucaria & Araucaria sp. & Araucariaceae \\
\hline Jacaranda & Jacaranda mimosifolia D. Don. & Bignoniaceae \\
\hline Nopal & Opuntia streptacantha Lem. & Cactaceae \\
\hline Cedro & Cupressus ssp. & Cupressaceae \\
\hline Pata de vaca & Bauhinia divaricata $\mathrm{L}$. & Fabaceae \\
\hline Colorín, Tzompancle & Erythrina coralloides DC. & Fabaceae \\
\hline Cajinicuil, vaina & Inga jinicuil Schltdl & Fabaceae \\
\hline Guaje & Leucaena esculenta (Moc. et Sessé ex DC.) Benth. & Fabaceae \\
\hline Aguacate criollo & Persea americana var. drymifolia (Schltdl. \&Cham) Blake & Lauraceae \\
\hline Aguacate & Persea americana Mill. var. hass & Lauraceae \\
\hline Nanche & Byrsonima crassifolia (L.) Kunth. & Malpighiaceae \\
\hline \multirow[t]{2}{*}{ Higo } & Ficus benjamina L. & Moraceae \\
\hline & Ficus carica $\mathrm{L}$. & Moraceae \\
\hline Amate amarillo & Ficus petiolaris Kunth & Moraceae \\
\hline Mora & Morus alba L. & Moraceae \\
\hline Guayabo & Psidium guajava L. & Myrtaceae \\
\hline Pomarosa & Syzygium jambos (L.) Alston & Myrtaceae \\
\hline Fresno & Fraxinus uhdei (Wenz.) Lingelsh. & Oleaceae \\
\hline Trueno & Ligustrum vulgare $\mathrm{L}$. & Oleaceae \\
\hline Pino & Pinus sp. & Pinaceae \\
\hline Granada & Punica granatum L. & Punicaceae \\
\hline Membrillo & Cydonia oblonga Mill. & Rosaceae \\
\hline Níspero & Eriobotrya japonica (Thunb.) Lindl. & Rosaceae \\
\hline Manzana & Prunus malus L. & Rosaceae \\
\hline Durazno & Prunus persica (L.) Batsch. & Rosaceae \\
\hline Lima, limón real y limón & Citrus aurantifolia (Christm.) Swingle & Rutaceae \\
\hline Naranja agria & Citrus aurantium $\mathrm{L}$. & Rutaceae \\
\hline Mandarina & Citrus reticulata Blanco & Rutaceae \\
\hline Naranja & Citrus sinensis (L.) Osbeck & Rutaceae \\
\hline Toronja & Citrus $x$ paradisi Macfad. & Rutaceae \\
\hline
\end{tabular}

fin, de los que se aprovechan los frutos como alimento (Tabla 2). Especies como Opuntia streptacantha Lem., Erythrina corallides DC, Yucca filifera Chabaud y Leucaena esculenta (Moc. et Sessé ex DC.) Benth., proporcionan follaje. Mientras que de E. corallides DC., Y. filifera Chabaud y L. esculenta (Moc. et Sessé ex DC.) Benth., se utilizan las flores como alimento, y de $O$ streptacantha Lem los cladodios. La disponibilidad de frutos y flores todo el año, beneficia a la familia al disponer de alimentos, además de que obtienen un ingreso económico por medio de su venta en el mercado municipal y en la comunidad. De Erythrina corallides DC. y Leucaena esculenta (Moc. et Sessé ex DC.) Benth se venden las flores como quelites.
Uso medicinal. Las especies medicinales en los huertos familiares de San Nicolás, son de gran importancia, existen 49 especies de árboles utilizadas en la medicina tradicional, que representan el 30 $\%$ de las especies presentes en los huertos. En cuanto a familias botánicas de uso medicinal por sus metabolitos secundarios destacan las Asteraceae, Fabaceae y Lamiaceae. En San Nicolás, los árboles como componente integral de los huertos familiares, son elementos multifuncionales, que proveen alimento y usos medicinales, para lo cual se usan hojas, flores o frutos, los cuales pueden estar disponibles todo el año.

Las especies arbóreas utilizadas en la medicina tradicional, se agruparon en 13 categorías de acuerdo con la clase y tipo de enfermedad que 


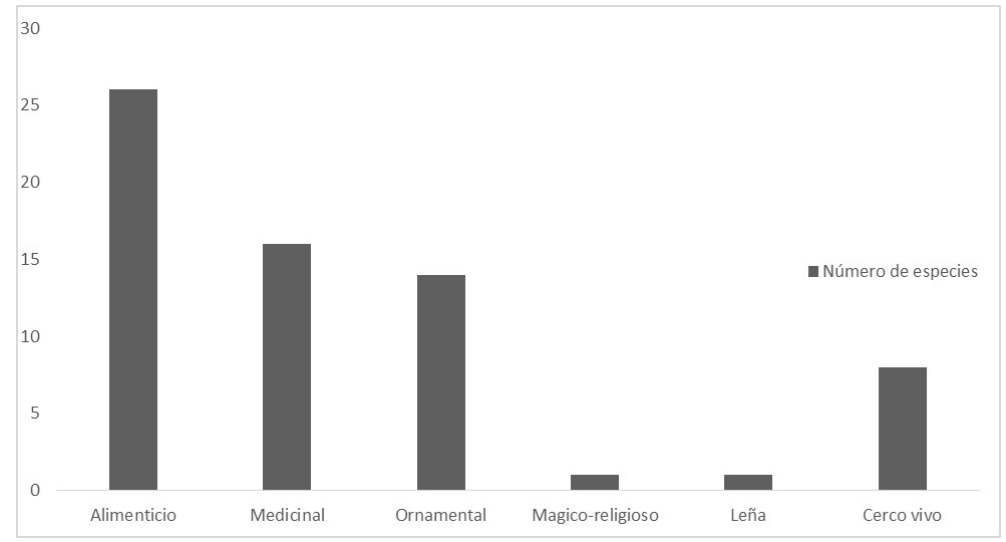

Figura 1. Principales usos del estrato arbóreo en los agroecosistemas huerto familiar.

Tabla 2. Especies arbóreas utilizadas como alimento, venta y parte usada, en los huertos familiares de la comunidad de San Nicolás, Estado de México.

\begin{tabular}{|c|c|c|c|c|c|c|}
\hline Nombre común & Nombre científico & Familia & Fruto & Flor & cladodios & Venta \\
\hline Yuca & Yucca filifera Chabaud & Agavaceae & & $\mathrm{X}$ & & \\
\hline Mango & Mangifera indica $\mathrm{L}$. & Anacardiaceae & $\mathrm{x}$ & & & $\mathrm{x}$ \\
\hline Ciruela criolla & pondias purpurea $\mathrm{L}$. & Anacardiaceae & $x$ & & & $\mathrm{x}$ \\
\hline Chirimoya & Annona cherimola Mill. & Annonaceae & $\mathrm{X}$ & & & $\mathrm{x}$ \\
\hline Anona & Annona reticulata $\mathrm{L}$. & Annonaceae & $x$ & & & $\mathrm{X}$ \\
\hline Nopal & Opuntia streptacantha Lem. & Cactaceae & $\mathrm{x}$ & & $\mathrm{X}$ & \\
\hline Tzompancle & Erythrina coralloides DC. & Fabaceae & & $x$ & & $x$ \\
\hline $\begin{array}{l}\text { Cajinicuil, } \\
\text { vaina }\end{array}$ & Inga jinicuil Schltdl & Fabaceae & $\mathrm{x}$ & & & $\mathrm{x}$ \\
\hline Guaje & $\begin{array}{l}\text { Leucaena esculenta (Moc. } \\
\text { et Sessé ex DC.) Benth. }\end{array}$ & Fabaceae & $\mathrm{x}$ & $\mathrm{x}$ & & $x$ \\
\hline Aguacate criollo & $\begin{array}{l}\text { Persea americana var. drymifolia (Schltdl. } \\
\& \text { Cham) Blake }\end{array}$ & Lauraceae & $\mathrm{x}$ & & & $\mathrm{X}$ \\
\hline $\begin{array}{l}\text { Aguacate } \\
\text { Nanche }\end{array}$ & $\begin{array}{l}\text { Persea americana Mill. var. hass } \\
\text { Byrsonima crassifolia (L.) }\end{array}$ & Lauraceae & $X$ & & & $\mathrm{X}$ \\
\hline & Kunth. & Malpighiaceae & $\mathrm{x}$ & & & \\
\hline Higo & Ficus carica $\mathrm{L}$. & Moraceae & $x$ & & & \\
\hline Mora & Morus alba L. & Moraceae & $\mathrm{X}$ & & & \\
\hline Guayabo & Psidium guajava L. Syzygium jambos (L.) & Myrtaceae & $x$ & & & $\mathrm{x}$ \\
\hline Pomarosa & Alston & Myrtaceae & $\mathrm{x}$ & & & $\mathrm{x}$ \\
\hline Granada & Punica granatum L. & Punicaceae & $\mathrm{X}$ & & & \\
\hline Membrillo & Cydonia oblonga Mill. & Rosaceae & $\mathrm{x}$ & & & \\
\hline Níspero & Eriobotrya japonica (Thunb.) Lindl. & Rosaceae & $\mathrm{X}$ & & & $\mathrm{X}$ \\
\hline Manzana & Prunus malus $\mathrm{L}$. & Rosaceae & $\mathrm{X}$ & & & \\
\hline Durazno & Prunus persica (L.) Batsch. & Rosaceae & $\mathrm{X}$ & & & \\
\hline $\begin{array}{l}\text { Lima, limón real } \\
\text { y limón }\end{array}$ & Citrus aurantifolia (Christm.) Swingle & Rutaceae & $\mathrm{X}$ & & & $x$ \\
\hline Naranja agria & Citrus aurantium L. & Rutaceae & $\mathrm{X}$ & & & \\
\hline Mandarina & Citrus reticulata Blanco & Rutaceae & $\mathrm{x}$ & & & \\
\hline Naranja & Citrus sinensis (L.) Osbeck & Rutaceae & $\mathrm{X}$ & & & $\mathrm{X}$ \\
\hline Toronja & Citrus $x$ paradisi Macfad. & Rutaceae & $x$ & & & \\
\hline
\end{tabular}

tratan. Los padecimientos más tratados con especies del estrato arbóreo son las enfermedades del aparato digestivo, para lo cual se utilizan 9 especies diferentes para padecimientos como dolor de estómago, diarreas y empacho; para esta clase de en- fermedades son utilizadas en infusión hojas, flores o frutos de Annona cherimola Mill., P. guajava L., B. crassifolia (L.) Kunth. y Punica granatum L., generalmente se usa una sola especie, no presentándose el uso de dos o más especies al preparar la 
infusión.

La diabetes en la actualidad es un padecimiento de importancia entre la población del país; lo anterior explica como éste padecimiento es uno de los más comunes, el cual es tratado con $O$. streptacantha Lem., E. japonica (Thunb.) Lindl. y $P$. americana Mil., en infusión de las hojas de aguacate, guayaba o cladodios del nopal. Mientras que los problemas de presión arterial se tratan con cladodios de $O$. streptacantha Lem., hojas de $E$. japonica (Thunb.) Lindl. y hoja y flores de $C$. sinensis (L.) Osbeck, en infusión.

Uso ornamental. El estrato arbóreo en los huertos familiares juega un papel estético, se identificaron 14 árboles con uso ornamental en los sistemas, pertenecientes a 13 géneros y 10 familias, donde las Fabaceae presentaron tres especies, y las Moraceae y Oleaceae dos especies cada una. Dentro de ésta categoría se encuentran 11 especies de angiospermas y tres de gimnospermas (Araucaria sp., Cupressus sp. y Pinus sp.) apreciadas principalmente por su follaje, a diferencia de las angiospermas cuyas flores son muy estimadas, por ejemplo, $Y$. filifera Chabaud, Thevetia thevetioides (H.B.K.) Schum. y Jacaranda mimosifolia D. Don. Estas especies arbóreas sirven también como soporte de otros organismos como las herbáceas que se ubican en macetas y enredaderas que embellecen el huerto familiar.

\section{Biogeografía y conservación}

El manejo y uso de los huertos familiares es dinámico, lo que conserva flora nativa, aunque también hay especies exógenas que satisfacen nuevas necesidades, por ejemplo árboles provenientes de Asia, Europa y América, por lo que 17 especies (47 $\%)$ son introducidas, 11 de ellas son asiáticas, con árboles frutales muy importantes en la economía como Mangifera indica L. Syzygium jambos (L.) Alston, E. japonica (Thunb.) Lindl. y cinco especies del género Citrus spp. Tres son europeas como Ligustrum sp. y 19 (52.7\%) nativas del Continente Americano, de éstas seis especies (31.5 $\%)$ se consideran originarias de México como $T$. thevetioides (H.B.K.) Schum. y O. streptacantha Lem., y 11 (57.8 \%) nativas como $P$. guajava L., $P$. americana Mill., I. jinicuil Schltdl. \& Cham. ex G. Don y L. esculenta (Moc. et Sessé ex DC.) Benth.

\section{DISCUSIÓN}

La biodiversidad del estrato arbóreo en los AHF, presenta especies representativas de las zonas tropicales y subtropicales. Se encontraron 36 especies, mucho menos especies que las especies reportadas para huertos familiares mayas, que posen una riqueza del estrato que supera en el doble a lo encontrado en el presente trabajo, por ejemplo De Clerck y Negreros-Castillo (2000) reportan 72 especies, 52 de ellas árboles frutales y 20 maderables (De la Rosa-Reyes et al. 2014, Juan y Madrigal 2005). Estas diferencias pueden deberse al proceso de fraccionamiento que están sufriendo los huertos familiares de San Nicolás, en donde los predios son utilizados para construir viviendas para los hijos mayores que se casan, o en algunos casos se substituye el huerto por una tienda $u$ otro negocio para apoyar el gasto familiar, lo que disminuirá la biodiversidad in situ actual de los AHF. La fragmentación del huerto para la construcción de casas, se ha observa en el estado de Puebla (Robichaux 1997) y en el estado de Yucatán (Rosado 2012). Lo que también coincide con Juan (2014) quien reporta que en la Región Sur del Estado de México, el espacio geográfico que ocupan los huertos familiares, se utiliza para construcción de viviendas o establecimientos comerciales. En México se observa disminución de la superficie de los huertos o en algunos casos desaparecen, lo que perjudica la soberanía alimentaria familiar y causa la pérdida de la biodiversidad de los huertos familiares (Mariaca et al. 2011).

La biodiversidad en estos sistemas está asociada con la presencia de especies clave o índice, en los AHF de San Nicolás, las especies P. americana Mill., Citrus spp., P. guajava L. E. japonica (Thunb.) Lindl. e I. jinicuil Schltdl, tiene amplia presencia en los huertos, lo que coincide con Gaytán et al. (2001) quienes indican que el aguacate ( $P$. americana Mill). y el níspero (E. japonica 
(Thunb.) Lindl). son dos de las especies clave que se encuentran con en mayor frecuencia en los huertos familiares, debido a su importancia como recurso alimentario para la familia y por su importancia comercial. Mientras que Alayón-Gamboa y Gurri-García (2007), Rebollar et al. (2008) y Van der Wal y Bongers (2013) en huertos tropicales indican que $P$. americana Mill., Citrus spp., P. guajava L, L. E. japonica (Thunb.) Lindl. e I. jinicuil Schltd son las especies más importantes, por aportar alimento e ingresos económicos por medio de su venta.

La estructura vertical de los AHF, está compuesta por tres estratos relacionados entre sí, que son arbóreo, arbustivo y herbáceo, estratos que coincide con los patrones encontrados en diversos huertos familiares (Gaytán et al. 2001, Lope-Alzina 2012, De la Rosa-Reyes et al. 2014. Aunque otros estudios reportan la presencia de seis estratos en huertos familiares mayas: herbáceo, arbustivo bajo, arbustivo alto, árboles frutales, árboles de madera y enredaderas (De Clerck y Negreros-Castillo 2000), riqueza que se relaciona no solo con condiciones socioculturales y económicas, si no también ambientales.

En los AHF de San Nicolás se tienen beneficios variados de las especies arbóreas como alimento, medicina u ornamento (Juan et al. 2007); siendo los más importantes para las familias de San Nicolás los de alimentos y ornamentales, además de que se dispone de excedentes que son vendidos en la comunidad o mercados, lo que permite un ingreso económico a la familia, tal como lo señalan Wezel y Bender (2003). Las condiciones ambientales, sociales, culturales y económicas influyen en las especies arbóreas que se conservan e introducen en el huerto, en el AHF se tienen las especies $P$. americana Mill. y $P$ guajava L., que tiene diversos usos, las cuales contribuyen al funcionamiento del huerto como agroecosistema, ya que su hojarasca contribuye al reciclaje de nutrimentos, para mantener la fertilidad del suelo.

Con la introducción de especies frutales exógenas, como M. indica L. Mil., Syzygium jambos (L.) Alston, Punica granatum L. y E. japonica
(Thunb.) Lindl. se satisfacen necesidades alimenticias y de medicina tradicional. En particular la introducción en el siglo XVI del género Citrus spp. a México (Cao 2003), el cual se ha convertido en una especie clave en los AHF. En los huertos del presente estudio se observa la presencia de cítricos en el $100 \%$, lo cual deja ver la importancia del género en la comunidad, como especie alimenticia para consumo familiar, para la venta como fruta y en la medicina tradicional donde se usan las hojas.

Los árboles, del estrato herbáceo y arbustivo, animales domésticos y silvestres, contribuyen a disponer de alimento diversos y otros bienes de uso durante todo el año en el AHF (González-Jácome 2012, González Jácome y Reyes 2014). El cultivo del huerto es un espacio estratégico para alcanzar la soberanía y seguridad alimentaria, porque sus productos son para autoconsumo o se venden para adquirir alimentos y otros satisfactores para la familia (Cano 2015). En San Nicolás, al conservar in situ, especies como $P$. americana Mill., de la que se cultivan tres variedades, la gente dispone de su propio germoplasma, por lo que no dependen de la compra de plántulas para el huerto. Estos procesos locales fortalecen la conservación del material genético de $P$. americana Mill., Citrus spp. y $P$. guajava L. y otras especies locales (Rosado 2012). A diferencia de sistemas agrícolas convencionales, los AHF son complejos, estables y multifuncionales, debido a que están integrados por plantas, animales, suelo y agua, en un área definida, cuya estructura, funcionamiento y manejo dependen de la organización familiar (Naira 2001, Albuquerquen et al. 2005, Juan et al. 2007).

La estructura, funcionamiento y manejo de los AHF, permite a las familias hacer un uso completo del estrato arbóreo como alimento, medicina, ornamento y sombra (Martínez y Juan 2005). Esta interacción entre el dosel arbóreo y el sotobosque, con el estrato arbustivo, es importante en los AHF, porque sirve para el cultivo del café, al proporcionarle sombra para su crecimiento y desarrollo. En el $36 \%$ de los AHF estudiados se encontró el cultivo del café, al respecto Martínez et al. (2007) mencionan que en cafetales de la Sierra Norte de 
Puebla la sombra está dada por especies de importancia comercial, como naranja, mandarina o plátano, que generan ingresos al productor.

\section{CONCLUSIONES}

El agroecosistema huerto familiar en la comunidad de San Nicolás, es un sistema complejo, estable y multifuncional, integrado por plantas, animales, suelo y agua, en un área definida; en donde la estructura, funcionamiento y manejo dependen de la organización familiar. La diversidad florística de especies arbóreas es alta, lo que ubica a estos sistemas como sitios de conservación de germoplasma in situ. La diversidad biológica del estrato arbóreo brinda bienes y servicios a las familias de la comunidad, como alimentos, medicinas e ingresos económicos. Las principales limitantes en estos espacios es la baja disponibilidad de agua, el abandono de la comunidad por la migración de los jóvenes, el fraccionamiento del espacio para la construcción de viviendas y los problemas fitosanitarios en árboles frutales.

\section{LITERATURA CITADA}

Alayón-Gamboa JA, Gurri-García F (2007) Home garden production and energetic sustainability in Calakmul, Campeche, México. Human Ecology 36: 395-407.

Albuquerque UP, Andrade LHC, Caballero J (2005) Structure and floristics of homegardens in northeastern Brazil. Journal of Arid Environments 62: 491-506.

Cano E (2015) Huertos familiares: un camino hacia la soberanía alimentaria. Pueblos y Fronteras 10: 70-91.

Cao LJ (2003) Reseña histórica acerca del ácaro del moho, Phyllocoptruta oleivora, plaga principal de los cítricos en Cuba. Fitosanidad 7: 67-74.

De Clerck KFAJ, Negreros-Castillo P (2000) Plant species of traditional Mayan homegardens of Mexico as analogs for multistrata agroforests. Agroforestry Systems 48: 303-317.

De la Rosa-Reyes PK, Vásquez-Dávila MA, Villegas-Aparicio Y, Jerez-Salas MP (2014) Los huertos familiares y la seguridad alimentaria de Cuilapam de Guerrero, Oaxaca, México. Revista Mexicana de Agroecosistemas 1: 40-51.

Gaytán AC, Vibrans H, Navarro HG, Jiménez M (2001) Manejo de huertos familiares periurbanos de San Miguel Tlaixpan, Texcoco, Estado de México. Boletín de la Sociedad Botánica de México 69: 39-62

Gliessman SR (2002) Agroecología: Procesos ecológicos en agricultura sostenible. Centro agronómico tropical de investigación y enseñanza. Litocat. Costa Rica. 359p.

Gliessman SR, Rosado-May FJ, Guadarrama-Zugasti C, Jedlicka J, Cohn A, Méndez VE, et al. (2007) Agroecología: promoviendo una transición hacia la sostenibilidad. Ecosistemas 16: 13-23.

Gillespie R, Knudson DM, Geilfus F (1993) The structure of four home gardens in the Petén, Guatemala. Agroforestry Systems 24: 157-170.

González-Jácome A (2012) Del huerto a los jardines y vecindades: procesos de cambio en un agroecosistema de origen antiguo. En: Mariaca MR (ed.). El huerto familiar del sureste de México. SERNAPAMECOSUR. Villahermosa, Tabasco. pp: 487-521.

González-Jácome A, Reyes L (2014) El conocimiento agrícola tradicional, la milpa y la alimentación: el caso del Valle de Ixtlahuaca, Estado de México. Geografía Agrícola 52/53: 21-42.

Gunnar G, Shivcharn SD, Daouda S (2004) Local use and cultural and economic value of products from trees in the parklands of the municipality of Cinzana, Mali. Economic Botany 58: 8-87. 
High C, Shackleton CM (2000) The comparative value of wild and domestic plants in home gardens of a South African rural village. Agroforestry Systems 48: 241-156.

Hylander K, Nemomissa S (2008) Home garden coffee as a repository of epiphyte biodiversity in Ethiopia. Frontiers in Ecology and the Environment 6: 524-528.

INEGI (2009) Resultados de XIII Censo Nacional de Población y Vivienda. Instituto Nacional de Estadística, Geografía e Informática. México, DF. http://www.inegi.org.mx/. Fecha de consulta 6 de agosto de 2015.

Jaramillo M, Castro M, Ruiz-Zapata T, Lastres M, Torrecilla P, Lapp M, et al. (2014) Estudio etnobotánico de plantas medicinales en la comunidad campesina de Pelelojo, municipio Urdaneta, Estado Aragua, Venezuela. Ernstia 24: 85-110.

Juan PJI, Madrigal DU (2005) Huertos, diversidad y alimentación en una zona de transición ecológica del estado de México. Ciencia Ergo Sum 2: 54-63.

Juan PJ I, Rebollar SR, Madrigal DU, Monroy JFG (2007) Huertos familiares en la región sur del Estado de México: funciones, importancia y manejo. En: Monrroy FG, Juan-Pérez JI, Carreto BF, Balderas PMA (eds.) Territorio, Agricultura y ambiente. Enfoques en el siglo XXI. UAEM. Estado de México. pp: 25-150.

Juan JIP (2014) Los huertos familiares en una provincia del subtrópico mexicano: análisis espacial, económico y sociocultural. 1er Edición. UAEM. Estado de México. 135p.

Kristensen M, Lykke AM (2003) Informant-based valuation of use and conservation preferences of savanna trees in Burkina Faso. Economic Botany 57: 203-217.

Kumar BM, Nair PKR (2004) The enigma of tropical homegardens. Agroforestry Systems 61: 135-152.

Lope-Alzina DG (2012) Avances y vacíos en la investigación en huertos familiares de la península de Yucatán. En: Mariaca MR (ed.) El huerto familiar del sureste de México. SERNAPAM-ECOSUR. Villahermosa, Tabasco pp: 9-109.

Lot A, Chiang F (1986) Manual de herbario. Consejo Nacional de la Flora de México. México, DF. 142p.

Magaña AM (2012) El huerto familiar en el sureste de México. En: Mariaca MR (ed.) El huerto familiar del sureste de México. SERNAPAM-ECOSUR. Villahermosa, Tabasco. pp: 176-195.

Mariaca R M (2012) La Complejidad del Huerto Familiar Maya del sureste de México. En: Mariaca MR (ed.) El huerto familiar del sureste de México. SERNAPAM-ECOSUR. Villahermosa, Tabasco. pp: 7-97.

Mariaca RM, González JA, Arias L (2011) El huerto maya yucateco en el siglo XVI. Península 6: 123-125.

Martínez B, Juan P (2005) Los huertos: una estrategia para la subsistencia de las familias campesinas. Annales de Antropología 39: 26-50.

Martínez M, Evangelista V, Basurto F, Mendoza M, Cruz-Rivas A (2007) Flora útil de los cafetales en la Sierra Norte de Puebla, México. Revista Mexicana de Biodiversidad 78: 15-40.

Moctezuma PS (2010) Una aproximación al estudio del sistema agrícola de huertos desde la antropología. Ciencia y Sociedad 35: 47-69.

Nair PKR (2001) Do tropical homegardens elude science, or is it the other way around. Agroforestry Systems 53: $239-245$.

Rebollar DS, Santos-Jiménez V, Tapia-Torres NA, Pérez-Olvera C (2008) Huertos familiares. Una experiencia en Chancah Veracruz, Quintana Roo. Polibotánica 25: 135-154. 
Robichaux D (1997) The stem family, residence rules and ultimogeniture in Tlaxcala and Mesoamerica. Ethnology 36: 149-171.

Rosado F (2012) Los huertos familiares, un sistema indispensable para la soberanía y suficiencia alimentaria en el sureste de México. En: Mariaca MR (ed.) El huerto familiar del sureste de México. SERNAPAMECOSUR. Villahermosa, Tabasco. pp: 350-521.

Tyler MG (1994) Ecología y medio ambiente. Grupo Editorial Iberoamericano. México. 867p.

Van der Wal H, Bongers F (2013) Biosocial and bionumerical diversity of variously sized home gardens in Tabasco, Mexico. Agroforestry Systems 87:93-07.

Wezel A, Bender S (2003) Plant species diversity of homegardens of Cuba and its significance for household food supply. Agroforestry Systems 57: 39-49.

White-Olascoaga L, Juan PJI, Chávez-Mejía X, Gutiérrez-Cedillo JG (2013) Flora Medicinal en San Nicolás, municipio de Malinalco, Estado de México. Polibotánica 35: 173-206. 